\title{
Paravertebral Block for Post-Operative Analgesia after Breast Cancer Surgery, Effects of Adding Morphine: Double Blind, Randomised Clinical Trial ${ }^{*}$
}

\author{
Gudmundur Björnsson $^{1 \#}$, Astridur Johannesdottir ${ }^{1}$, Bjarni Valtysson ${ }^{2}$, Gisli H. Sigurdsson ${ }^{1,3}$ \\ ${ }^{1}$ Department of Anaesthesiology and Intensive Care, Landspitali University Hospital, Reykjavik, Iceland; ${ }^{2}$ Orkuhúsid Clinic, Rey- \\ kjavik, Iceland; ${ }^{3}$ Faculty of Medicine, University of Iceland, Reykjavik, Iceland. \\ Email:" gudmbjor@landspitali.is
}

Received February $3^{\text {rd }}, 2013$; revised March $11^{\text {th }}, 2013$; accepted March $17^{\text {th }}, 2013$

\begin{abstract}
Introduction: Thoracic paravertebral block (PVB) block is frequently used in breast cancer surgery for postoperative pain management. Adding opioids to local anaesthetics has been shown to have beneficial effects during epidural analgesia. Our hypothesis was that adding morphine to bupivacaine for PVB would improve analgesia provided by this procedure. Methods: 60 patients ( 25 - 75 years) undergoing elective surgery for breast cancer were randomly assigned to one of two groups. Both groups received a single injection thoracic paravertebral block; group BAM with $20 \mathrm{ml} 0.5 \%$ bupiva- caine, epinephrine and morphine while group BA received identical block except morphine was given subcutaneously. All patients had general anaesthesia. Results: Severity of pain and nausea was low in both groups. Pain scores remained below 20/100 after the first 2 hours throughout the 72 hours of the study. There was no significant difference between the groups in pain scores, consumption of additional morphine or nausea scores. Shoulder mobility was also very good in both groups. Conclusion: Thoracic paravertebral block with bupivacaine and epinephrine was associated with good postoperative analgesic effects and low incidence of nausea and vomiting. The addition of morphine to the local anaesthetic solution in paravertebral block did not have any additional analgesic effects.
\end{abstract}

Keywords: Thoracic Paravertebral Block; Bupivacaine; Epinephrine; Morphine; Breast Cancer

\section{Introduction}

Traditionally breast cancer surgery has been performed under general anaesthesia. However, it has been associated with troublesome postoperative pain as well as high incidence of nausea and vomiting. During recent years paravertebral nerve block has been suggested as an attractive alternative for this type of surgery, providing adequate pain relief and a reduced incidence of postoperative nausea and vomiting [1-3].

In most clinical trials on paravertebral nerve block the local anaesthetics bupivacaine or ropivacaine have been used alone [1-5]. During postoperative pain management with continuous epidural analgesia, however, addition of epinephrine and opioids has been shown to significantly improve intensity of the block [6-9].

Our hypothesis was that adding morphine to bupivacaine and epinephrine would improve the quality of paravertebral blockade during breast cancer surgery. The

\footnotetext{
*Conflict of interest: None of the authors have any conflict of interest concerning this study.

"Corresponding author.
}

effect on postoperative pain intensity and morphine consumption was studied as a primary outcome. Nausea and vomiting and number of patients receiving antiemetic medication as well as hospital stay were secondary outcome measures.

\section{Methods}

After institutional ethics committee approval of the study protocol and written informed consent, 60 patients (ASA I-II), aged 25 to 75 years, undergoing elective surgery for cancer of the breast were entered into this prospective double blind clinical trial. They underwent either unilateral wedge resection with exploration of the axilla or unilateral mastectomy with exploration of the axilla. The minimum hospital follow-up time was 24 hours. Patient exclusion criteria included coagulation disorder, infection at the site of blockade and a history of adverse reaction to local anaesthetics or morphine, morbid obesity, current medication with opioids, age over 75 years, significant cardiopulmonary disease, and history of severe PONV. 
Patients were randomly assigned into one of two groups. Group BA received thoracic paravertebral block (PVB) with a single injection at T3 using $20 \mathrm{ml} 0.5 \%$ bupivacaine, epinephrine $5 \mu \mathrm{g} / \mathrm{ml}$ and $0.4 \mathrm{ml}$ isotonic saline. In addition they received a subcutaneous injection of morphine $10 \mathrm{mg} / \mathrm{ml} 0.4 \mathrm{ml}$. The other group (group BAM) received PVB using $20 \mathrm{ml} 0.5 \%$ bupivacaine and fresh epinephrine $5 \mu \mathrm{g} / \mathrm{ml}$ as in group BA, but unlike group BA, group BAM also received paravertebral morphine, $0.4 \mathrm{ml}(10 \mathrm{mg} / \mathrm{ml})$ and a subcutaneous injection of isotonic saline $0.4 \mathrm{ml}$.

Before surgery the patients were enrolled to one of two groups using a list of random numbers, generated by a computer, in sealed envelopes. The randomization process was in the hands of pharmacists of the hospital pharmacy who provided the study drugs in syringes ready for injection. Two experienced anaesthesiologists performed all the blocks. The physician providing the PVB was blinded to the content of the local anaesthetic and subcutaneous solutions. Before performing the PVB the patients were sedated in a sitting position by administering $1.0-2.5 \mathrm{mg}$ of midazolam intravenously (i.v.). The block was performed using the technique described by Eason and Wyatt [10] using a 25 gauge, $9 \mathrm{~cm}$ Quincke spinal needle (Braun, Spinocan, Melsungen, Germany). The spinal needle with a depth marker was introduced at $90^{\circ}$ to the skin in all planes $2.5 \mathrm{~cm}$ lateral to the anatomical midline at the cephalad end of the spinous process of the 3rd thoracic vertebra and advanced to strike the rib or transverse process of the vertebra below. When bone contact was reached, the needle was withdrawn to the subcutaneous tissue and redirected cephalad to pass above the transverse process or the rib and then advanced further $1.0-1.5 \mathrm{~cm}$. After careful aspiration the $20.5 \mathrm{ml}$ of drug mixture (bupivacaine, epinephrine and isotonic saline in group BA and bupivacaine, epinephrine and morphine in group BAM) was administered into the paravertebral space. Injection time was 1 - 2 minutes. Efficacy of the PVB was assessed by loss of sensibility to cold using ethyl chloride spray and to pin prick over the involved dermatomes at least 10 min after completion of the block.

Following completion of the blockade all patients received general anaesthesia with propofol $2-3 \mathrm{mg} / \mathrm{kg}$ i.v. and fentanyl $0.3-0.6 \mu \mathrm{g} / \mathrm{kg}$ i.v. A laryngeal mask airway (LMA) was inserted and anaesthesia was maintained with sevoflurane and nitrous oxide in oxygen and spontaneous breathing. Fentanyl $0.3-0.6 \mu \mathrm{g} / \mathrm{kg}$ was administered as deemed necessary by the attending anaesthesiologist. All patients received paracetamol $1 \mathrm{~g}$ rectally immediately after start of general anaesthesia and postoperatively $1 \mathrm{~g}$ of paracetamol orally or rectally every six hours for at least $24 \mathrm{~h}$. All patients were treated with on- dansetron $4 \mathrm{mg}$ i.v. about $20 \mathrm{~min}$ before the end of the operation for prevention of nausea.

Procedural data were recorded including time for placement of PVB, start and end of general anaesthesia as well as the surgical procedure. The total intraoperative fentanyl dose administered to each patient was recorded.

In the postoperative care unit all patients were connected to an i.v. PCA pump containing morphine with a dose of $1 \mathrm{mg}$, with $10 \mathrm{~min}$ lockout period. Nurses in the postoperative care unit were instructed to give rescue doses of morphine, $2 \mathrm{mg}$ i.v. as they deemed necessary.

Visual analog scale (VAS) pain scores were recorded at $30 \mathrm{~min}, 60 \mathrm{~min}, 2 \mathrm{~h}, 6 \mathrm{~h}, 24 \mathrm{~h}, 48 \mathrm{~h}$ and $72 \mathrm{~h}$ on a $100-$ $\mathrm{mm}$ scale $(0 \mathrm{~mm}=$ no pain to $100 \mathrm{~mm}=$ worst pain imaginable). Nausea was measured by a comparable VAS ( 0 $\mathrm{mm}=$ no nausea to $100 \mathrm{~mm}=$ vomiting) at the same time points. Ondansetron $4 \mathrm{mg}$ i.v. was administered whenever a patient's nausea score was higher than $30 \mathrm{~mm}$. Patients that were discharged from the hospital recorded their scores on standardized forms with two $100 \mathrm{~mm}$ visual analog scales at 48 and $72 \mathrm{~h}$ and submitted by mail. Postoperative painful restricted movement of arm, classified into three grades (restricted, fair and free; free meaning able to put the hand behind the head and fair meaning abduction to 90 degrees without discomfort) was recorded at $30 \mathrm{~min}, 60 \mathrm{~min}, 2 \mathrm{~h}, 6 \mathrm{~h}$ and $24 \mathrm{~h}$. Postoperative assessments were made by an observer blinded to the study medication (i.e. local anaesthetic/morphine solution and peroperative analgesic requirements). Postoperative PCA consumption of morphine was recorded as well as antiemetic requirements. Hospital stay was measured from the time of arrival to the recovery room until the time when the patient left the hospital, which was decided by the surgeon in charge.

Descriptive statistics for demographics, procedural data and intraoperative opioid administration were produced. Pain and nausea scores, restriction of movement as well as PCA morphine consumption and antiemetic requirements were analysed. In a pilot study the response within each subject group was normally distributed with standard deviation of 12 . We decided that a clinically significant difference in the experimental and control mean pain scores would be 10. Sample size calculation based on that suggested we would need to study 24 experimental subjects and 24 control subjects to be able to reject the null hypothesis that the population means of the experimental and control groups are equal with probability (power) 0.8 [11]. The Type I error probability associated with this test of this null hypothesis is 0.05 .

We decided to include 60 patients (30 in each group) to have a margin for uncertainty of the estimated standard deviation and possible dropouts. A two-tailed t-test was used for comparison of the two groups. 


\section{Results}

54 women completed the whole study protocol. Two were excluded, one because of error in the preparation of the local anaesthetic and the other because the operation was terminated as a result of unexpected histopathologic finding. One patient had a reoperation at 32 hours due to bleeding that started between 6 and 24 hours and only the scores for the first 6 hours were used. Three did not return the form for pain and nausea score at 48 and 72 hours. Their scores for the first 24 hours were entered in the statistical analysis. Thus values from 58 patients were analysed (Figure 1). During general anaesthesia low systolic blood pressure was often registered requiring occasional intravenous doses of ephedrine but no serious side effects of the paravertebral block were noted in any of the patients throughout the study. Preoperative assessments of the block revealed that there was considerable variation in spread of anaesthesia but none was judged as failed block. The number of blocked segments in each group is shown in Table 1.

31 patients received PVB with bupivacaine, epinephrine and morphine and a subcutaneous injection of saline (BAM) while 29 patients received PVB with bupivacaine and epinephrine and a subcutaneous injection of morphine (BA).

Analysis of demographic data revealed that the study groups were identical (Table 2). The ratio between mastectomy and wedge resection was $15 / 16$ respectively in the BAM group and $11 / 16$ in the BA group.

After the first two hours the VAS pain scores remained below $20 \mathrm{~mm}$ and there was no significant difference in

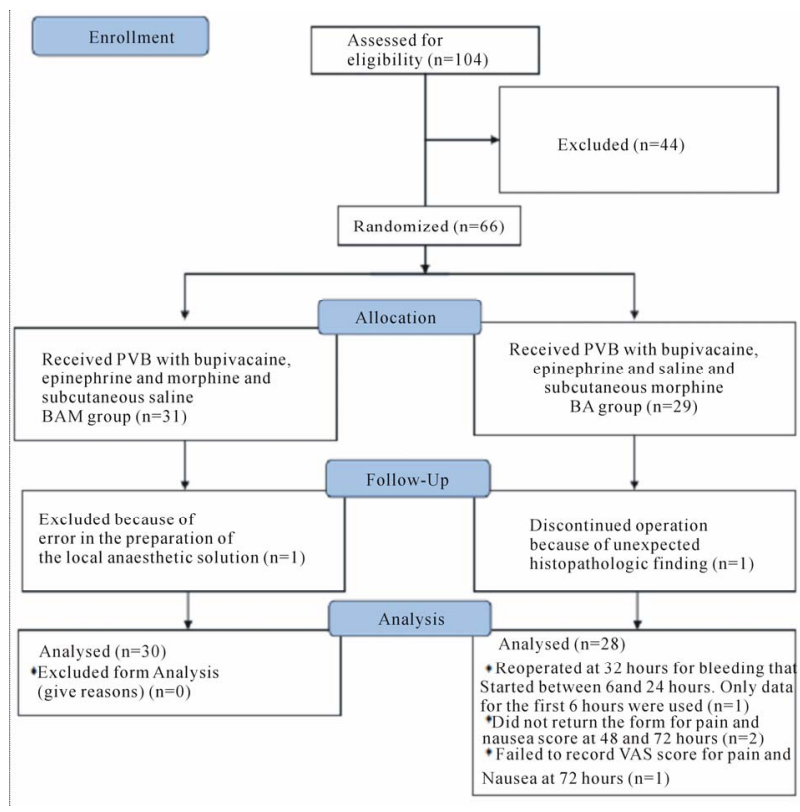

Figure 1. Enrollment of patients. pain score between the two main groups (Figure 2). The amount of fentanyl given during the operation was equal in both groups. There was no difference in morphine consumption between the groups, neither PCA administered nor total consumption (Table 3).

The nausea scores on the predefined time points for observation were low and no significant difference in nausea score between the groups (Figure 3). Only 4 out of 31 in the BAM group and 1 out of 27 in the BA group scored $30 / 100 \mathrm{~mm}$ or higher on the visual analog scale for nausea at some time point during the observation period. However, seven patients in the BAM group received antiemetic medication. Sometime during the first 24 hours postoperatively compared with 12 patients in the BA group (ns).

Shoulder mobility was excellent, i.e. free, in at least $50 \%$ of patients in the BAM group throughout the first 24 hours postoperatively (Figure 4(a)). In the BA group this fraction was between $40 \%$ and $50 \%$ at $30 \mathrm{~min}$ and 6

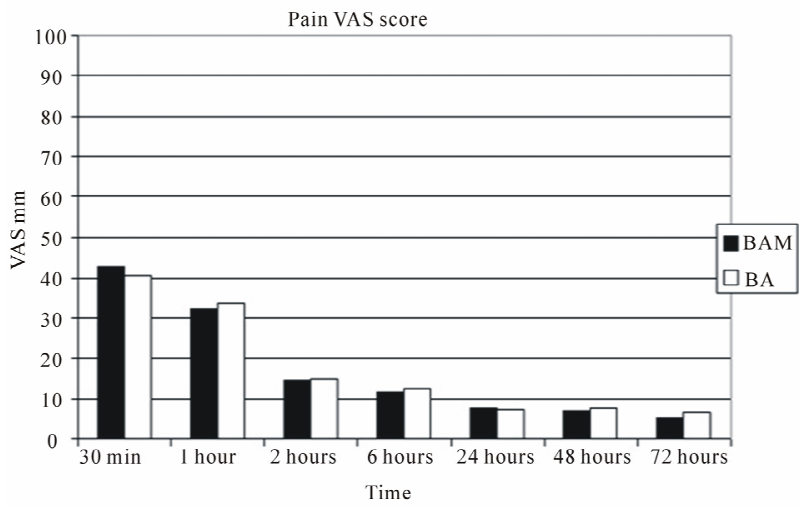

Figure 2. Comparison of average visual analog pain scores during the first 72 hours postoperatively. Group BAM received bupivacaine, epinephrine and morphine into the paravertebral space and subcutaneous saline. Group BA received bupivacaine, epinephrine and saline into the paravertebral space and subcutaneous morphine. There was no statistical difference between the groups. Observe that from two hours postoperatively the pain score remained below 20/100 throughout the study.

Table 1. Number of blocked segments.

\begin{tabular}{lcc}
\hline \multicolumn{1}{c}{ Group } & BAM & BA \\
\hline $\begin{array}{l}\text { Number of anaesthetized } \\
\text { segments, cold, median (range). }\end{array}$ & $6(0-15)$ & $7(4-14)$ N.S. \\
$\begin{array}{l}\text { Number of anaesthetized } \\
\text { segments, pinprick median (range). }\end{array}$ & $4(2-10)$ & $5(2-11)$ N.S. \\
\hline
\end{tabular}

Assessment of sensibility to cold and pinprick. Group BAM received paravertebral block with a solution containing bupivacaine, epinephrine and morphine while group BA got paravertebral block with bupivacaine and epinephrine and subcutaneous morphine. There was no significant difference in number of blocked segments. 
Table 2. Demographic characteristics of 58 patients included in data analysis.

\begin{tabular}{lcc}
\hline Variable/groups & BAM & BA \\
\hline Age (year; mean \pm SD) & $54.6 \pm 10.1$ & $53.5 \pm 11.0$ \\
Height (cm; mean \pm SD) & $166.6 \pm 4.5$ & $167.8 \pm 5.0$ \\
Weight $(\mathrm{kg}$; mean $\pm \mathrm{SD})$ & $71.8 \pm 12.4$ & $72.4 \pm 10.7$ \\
$\begin{array}{l}\text { Surgical procedure mastectomy } \\
\text { and axillaryexploration, number }\end{array}$ & 15 & 11 \\
$\begin{array}{l}\text { Wedge resection and axillary } \\
\text { exploration, number }\end{array}$ & 16 & 16
\end{tabular}

exploration, number

Duration of surgery

$(\mathrm{min} ;$ mean $\pm \mathrm{SD})$

$79.9 \pm 15.3 \quad 80.5 \pm 20.1$

Group BAM received paravertebral block with a solution containing bupivacaine, epinephrine and morphine while group BA got paravertebral block with bupivacaine and epinephrine and subcutaneous morphine. There was no statistical difference between the groups in any of the parameters presented.

Table 3. Morphine consumption in the first 24 hours.

\begin{tabular}{|c|c|c|}
\hline Variable/group & BAM & $\mathrm{BA}$ \\
\hline $\begin{array}{l}\text { PCA morphine consumption, } \\
\text { mg; mean } \pm \text { SD }\end{array}$ & $14.8 \pm 11.2$ & $13.7 \pm 10.4$ \\
\hline $\begin{array}{l}\text { Total morphine } \\
\text { consumption, mg; mean } \pm \text { SD }\end{array}$ & $16.9 \pm 12.8$ & $16.1 \pm 12.6$ \\
\hline $\begin{array}{c}\text { Peroperative fentanyl } \\
\text { administration, } \mu \mathrm{g} ; \text { mean } \pm \mathrm{SD}\end{array}$ & $83.9 \pm 34.3$ & $77.4 \pm 32.6$ \\
\hline
\end{tabular}

Group BAM received paravertebral block with a solution containing bupivacaine, epinephrine and morphine while group BA got paravertebral block with bupivacaine and epinephrine and subcutaneous morphine. Total morphine consumption represents PCA morphine consumption plus nurse controlled rescue doses. There was no statistical difference between the groups in any of the parameters.

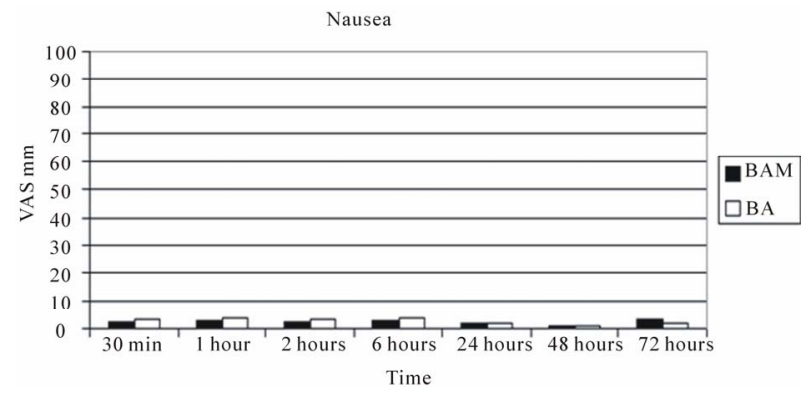

Figure 3. VAS scores for nausea. Group BAM received bupivacaine, epinephrine and morphine into the paravertebral space and subcutaneous saline. Group BA received bupivacaine, epinephrine and saline into the paravertebral space and subcutaneous morphine. 7 out of 30 patients in the BAM group received antiemetic postoperatively compared with 12 out of 27 in the BA group.

hours and at the other time points it was $50 \%$ or greater (Figure 4(b)). Patients assessed as having free or fair mobility at any of the given time points were $\geq 90 \%$ in both groups.

The mean hospital stay for both groups was $47 \pm 14.7$ hours and there was no significant difference between the groups (Table 4).

\section{Discussion}

The results of this study do not confirm the hypothesis, that adding morphine to bupivacaine for paravertebral block (PVB) would improve the analgesia provided by this procedure. However, the pain scores were very low in both groups after the first 2 hours. In fact they did remain below $20 \mathrm{~mm}$ throughout the 72 hours of the study, which is generally accepted as very good. The PONV score was also good in both groups as well as sholder

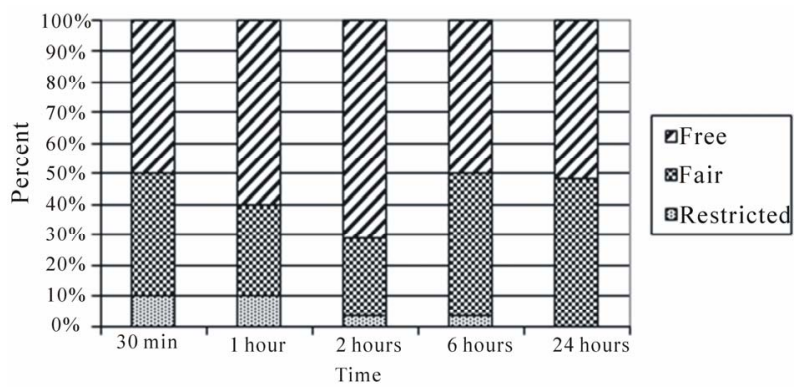

(a)

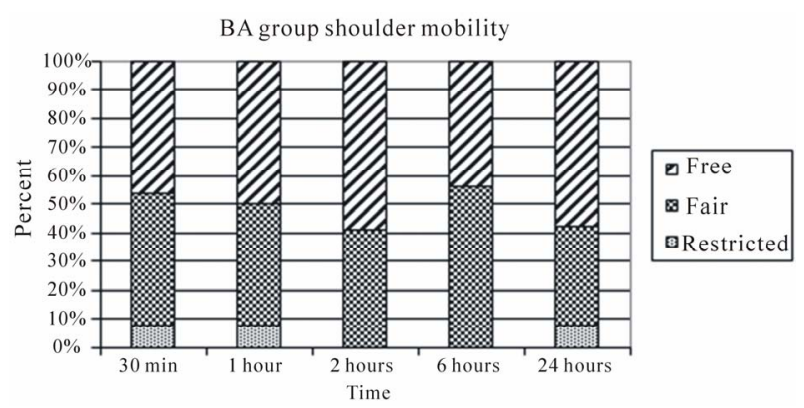

(b)

Figure 4. (a) Estimated restriction of movement in the shoulder joint by pain classified into three grades (restricted, fair and free; free meaning able to put the hand behind the head and fair meaning abduction to 90 degrees without discomfort) in the group receiving paravertebral bupivacaine, epinephrine and morphine and subcutaneous saline. (b) Estimated restriction of movement in the shoulder joint by pain classified into three grades (restricted, fair and free; fair meaning abduction to 90 degrees without discomfort) in the group receiving paravertebral bupivacaine, epinephrine and saline and subcutaneous morphine.

Table 4. Hospital stay (hours).

\begin{tabular}{ccccc}
\hline Group & N & Mean \pm SD & Minimum & Maximum \\
\hline BA & 27 & $49 \pm 16$ & 22 & 100 \\
BAM & 31 & $46 \pm 11$ & 20 & 74 \\
Total & 58 & $47 \pm 13$ & 20 & 100 \\
\hline
\end{tabular}

Group BA received paravertebral block with bupivacaine and epinephrine and subcutaneous morphine while the BAM group received paravertebral block with bupivacaine, epinephrine and morphine. $\mathrm{SD}=$ Standard deviation. There was no statistical difference in hospital stay between the groups. 
mobility. These results suggest that paravertebral block with bupivacaine and epinephrine, with or without morphine offers efficient postoperative pain relief after breast cancer sugery.

In previous studies the efficiency of PVB in providing analgesia after surgery for cancer of the breast has been shown to be superior to traditional analgesic regimens. Therefore the present study was designed to have only two groups i.e. a treatment group and a control group but not a second control group with a sham PVB. Although techniques that employ multiple segmental blocks are known to give more complete analgesia than a single injection approach we did not consider it worthwhile or even ethical to put our patients through such an extensive procedure for postoperative analgesia bearing in mind that all were going to have general anaesthesia nonetheless.

The VAS pain scores were similar in both groups as was morphine consumption. The PCA dose of $1 \mathrm{mg}$ morphine with a lockout period of $10 \mathrm{~min}$ proved to be too small for many patients in the first postoperative hour and nurse controlled rescue doses had to be given during that time but rarely later. In general the pain scores and opioid consumption in this study population was low. One might argue that using $0.5 \%$ bupivacaine produced too powerful analgesia for the possible subtle effect of morphine to be detected. However, using a weaker solution on those thoracal segments would not have brought any advantage. With the long effect of perispinal morphine in mind one could speculate that a difference in analgesia might be seen when the effect of the local anesthetic was waning. This was not the case.

The pain scores were very low in both goups after the first 2 hours and we did not see a tendency to a difference between the groups at any time. It is therefore unlikely that a larger sample size would have detected a clinically significant difference provided the same methods protocol was used.

The decision to exclude patients with history of extreme PONV was made after encountering such patients in a pilot study where it proved to be extremely difficult to obtain reliable pain scores. We were mainly interested in finding out if the paravertebral route of administration for morphine was associated with more nausea than the subcutaneous route. The incidence of significant nausea turned out to be low in both groups. More patients in the BAM group scored $30 / 100 \mathrm{~mm}$ or higher for nausea at some time point compared to the patients of the BA group who on the other hand received more doses of antiemetic drugs. We can state that the paravertebral route of administration of morphine was not associated with increased nausea.

We did not expect to see a difference in shoulder mobility on the grounds that opioids are mainly effective for pain at rest and less so for pain on movement. Our results agree with that as shoulder mobility was fair to excellent in the vast majority of patients in both groups.

Epinephrine has been shown to increase sensory block and improve the analgesic effect of a mixture og bupivacaine and fentanyl when given epidurally [7]. The influence of epinephrine on the pain relieving effect of perispinal morphine has been more equivocal. We decided to include epinephrine in order to delay the uptake of morphine into the systemic circulation and thus facilitate penetration into the spinal nerves.

In conclusion thoracic paravertebral block with bupivacain and epinephrine appears to be safe and efficient way of providing postoperative analgesia for breast cancer surgery. Adding $4 \mathrm{mg}$ of morphine to the local anaesthetic solution does not bring additional benefit but does not seem to do any harm either. It does not affect nausea or duration of hospital stay.

\section{Acknowledgements}

This study was supported by grant from the Landspitali University Hospital Research Fund.

Special thanks to the hospital pharmacy staff for their contribution in completing this study and in particular to the pharmacists Hulda Hardardóttir and Elín I. Jacobsen for their help in setting up the study protocol.

\section{REFERENCES}

[1] F. Pusch, H. Freitag, C. Weinstabl, R. Obwegeser, E. Huber and E. Wildling, "Single-Injection Paravertebral Block Compared to General Anaesthesia in Breast Surgery," Acta Anaesthesiologica Scandinavia, Vol. 43, No. 7, 1999, pp. 770-774. doi:10.1034/j.1399-6576.1999.430714.x

[2] M. M. Najarian, J. M. Johnson, J. Landercasper, P. Havlik, P. J. Lambert and D. McCarthy, "Paravertebral Block: An Alternative to General Anesthesia in Breast Cancer Surgery," American Surgery, Vol. 69, No. 3, 2003, pp. 213-218.

[3] P. M. Kairaluoma, M. S. Bachmann, A. K. Korpinen, P. H. Rosenberg and P. J. Pere, "Single-Injection Paravertebral Block before General Anesthesia Enhances Analgesia after Breast Cancer Surgery with and without Associated Lymph Node Biopsy," Anesthesia \& Analgesia, Vol. 99, No. 6, 2004, pp. 1837-1843. doi:10.1213/01.ANE.0000136775.15566.87

[4] J. F. Moller, L. Nikolajsen, S. A. Rodt, H. Ronning and P. S. Carlsson, "Thoracic Paravertebral Block for Breast Cancer Surgery: A Randomized Double-Blind Study," Anesthesia \& Analgesia, Vol. 105, No. 6, 2007, pp. 1848-1851. doi:10.1213/01.ane.0000286135.21333.fd

[5] G. Hura, P. Knapik, H. Misiołek, A. Krakus and J. Karpe, "Sensory Blockade after Thoracic Paravertebral Injection of Ropivacaine or Bupivacaine," European Journal of 
Anaesthesiology, Vol. 23, No. 8, 2006, pp. 658-664. doi:10.1017/S0265021506000561

[6] G. Niemi and H. Breivik, "Epidural Fentanyl Markedly Improves Thoracic Epidural Analgesia in a Low-Dose Infusion of Bupivacaine, Adrenaline and Fentanyl. A Randomized, Double-Blind Crossover Study with and without Fentanyl," Acta Anaesthesiologica Scandinavia, Vol. 45, No. 2, 2001, pp. 221-232. doi:10.1034/j.1399-6576.2001.450214.x

[7] G. Niemi and H. Breivik, "Adrenaline Markedly Improves Thoracic Epidural Analgesia Produced by a Low-Dose Infusion of Bupivacaine, Fentanyl and Adrenaline after Major Surgery. A Randomised, Double-Blind, Cross-Over Study with and without Adrenaline," Acta Anaesthesiologica Scandinavia, Vol. 42, No. 8, 1998, pp. 897-909. doi:10.1111/j.1399-6576.1998.tb05348.x

[8] G. Niemi and H. Breivik, "Epinephrine Markedly Improves Thoracic Epidural Analgesia Produced by a Small-Dose Infusion of Ropivacaine, Fentanyl, and Epinephrine after
Major Thoracic or Abdominal Surgery: A Randomized, Double-Blinded Crossover Study with and without Epinephrine," Anesthesia \& Analgesia, Vol. 94, No. 6, 2002, pp. 1598-1605.

[9] G. Niemi and H. Breivik, "The Minimally Effective Concentration of Adrenaline in a Low-Concentration Thoracic Epidural Analgesic Infusion of Bupivacaine, Fentanyl and Adrenaline after Major Surgery. A Randomized, Double-Blind, Dose-Finding Study," Acta Anaesthesiologica Scandinavia, Vol. 47, No. 4, 2003, pp. 439-450. doi:10.1034/j.1399-6576.2003.00077.x

[10] M. J. Eason and R. Wyatt, "Paravertebral Thoracic BlockA Reappraisal," Anaesthesia, Vol. 34, No. 7, 1979, pp. 638-642. doi:10.1111/j.1365-2044.1979.tb06363.x

[11] W. D. Dupont and W. D. Plummer Jr., "Power and Sample Size Calculations for Studies Involving Linear Regression," Controlled Clinical Trials, Vol. 19, No. 6, 1998, pp. 589-601. doi:10.1016/S0197-2456(98)00037-3 